\title{
Uncertainty Estimation of a CFD-Methodology for the Performance Analysis of a Collective and Cyclic Pitch Propeller
}

\author{
A. Dubois, Z. Q. Leong, H. D. Nguyen and J. R. Binns \\ Australian Maritime College, University of Tasmania \\ 1 Maritime Way, 7248 Newnham, Launceston, Tasmania, Australia
}

\begin{abstract}
Estimation and analysis of the uncertainty introduced by using a numerical model for the investigation and study of any type of flow problem have become common industry practice. Through understanding and evaluation of the uncertainty introduced by a numerical model, the accuracy and applicability of the model itself are evaluated. In this paper, the numerical uncertainty of a CFD-methodology developed to analyse the hydrodynamic performance of a collective and cyclic pitch propeller (CCPP) is estimated and analysed. The CCPP is a novel propulsion and manoeuvring concept for autonomous underwater vehicles, aimed to generate both propulsion and manoeuvring forces through advanced control of the propeller's blade pitch. The numerical uncertainty is established for three performance parameters, the generated propulsive force, the side-force magnitude, and the side-force orientation, by conducting a grid and time-step refinement study over three operational conditions. Additionally, the influence of the oscillatory uncertainty, introduced by the periodic nature of the problem, is investigated although shown to have a minimal effect when properly monitored. Based on a least-squares regression analysis of the refined simulation results, the numerical uncertainty is proven to be dominated by the introduced discretisation errors. In the case of the propulsive and side-force magnitude, the total uncertainty is dictated by the time discretisation uncertainty under bollard pull conditions, while the total uncertainty of the captive cases is mainly a result of the spatial discretisation uncertainty. The total uncertainty in the side-force orientation is observed to be primarily a consequence of the time discretisation uncertainty for all simulated cases. Overall, the
\end{abstract}


total uncertainty for captive cases can be considered satisfactory for all three performance parameters, while further work is needed to reduce the observed uncertainty of the simulations under bollard pull conditions.

Keywords:

Numerical uncertainty, CFD, Collective and cyclic pitch propeller

1 Nomenclature

\begin{tabular}{ll}
$c$ & Chord length \\
$u$ & Flow velocity \\
$h_{i}$ & Discretisation parameter \\
$n$ & Rotational CCPP velocity \\
$n_{c} / n_{r} / n_{s}$ & Chord / radius / span - based cell count \\
$n_{\text {osc }}$ & Number of oscillation cycle \\
$n_{s t}$ & Number of spatial / time discretisation levels \\
& \\
$D$ & Propeller diameter \\
$F_{s}$ & Resulting side-force \\
$F_{s}$ & Side-force magnitude \\
$F_{p r}$ & Propulsive force \\
$F_{s}^{i n t}$ & Intended side-force \\
$F_{s}^{\text {per }}$ & Perpendicular side-force \\
$S F$ & Safety factor \\
$T$ & Pitch period \\
$T^{*}$ & Dimensionless time \\
$U$ & Uncertainty \\
$\Gamma$ & \\
\hline &
\end{tabular}




$\begin{array}{ll}\epsilon & \text { Discretisation error } \\ \kappa & \text { Error estimation constant } \\ \Delta \Gamma_{f i t} & \text { Difference fitted and actual result } \\ \Delta \Gamma_{\text {min }} & \text { Difference arbitrary and finest result } \\ \Pi_{\left(\phi_{a z i}\right)} & \text { Azimuthal pitch profile } \\ \Pi_{c o l l} & \text { Collective pitch } \\ \Pi_{c y c l}^{\uparrow} / \Pi_{c y c l}^{\leftrightarrow} & \text { Cyclic pitch } \\ \phi_{a z i} & \text { Azimuthal blade position } \\ \phi_{s} & \text { Side-force orientation } \\ \sigma & \text { Standard deviation }\end{array}$

\section{Introduction}

In recent decades Autonomous Underwater Vehicles (AUVs) have become an essential part of underwater missions in many industries and fields of research [1]. AUVs are deployed to remote underwater locations, often travelling over long distances to survey, recover lost objects, investigate off-shore structures or marine life, and are even deployed for underwater surveillance missions. Traditional AUV propulsion and manoeuvring systems do not offer the flexibility of combined long-range efficiency to travel from one site to another with low-speed manoeuvring capabilities once arrived at the site of interest. The collective and cyclic pitch propeller (CCPP) is a novel system to both propel and manoeuvre AUVs. Through advanced control of the propeller's blade pitch, the CCPP enables an AUV to efficiently travel over long-distances, while offering effective manoeuvring at low speeds to execute specific parts of its predefined mission.

The CCPP combines two distinct pitch control concepts, thereby enabling precise management of the orientation or pitch of the propeller blades. Collective pitch governs the simultaneous pitch of all propeller blades and is a widely used concept in the maritime industry. The collective pitch control allows to match the propulsion system to various desired operational conditions, to optimise the propulsive efficiency, and even to change the propulsion 
direction [2]. Through cyclic pitch control, the pitch of each blade is changed individually over the azimuthal cycle of the propeller. The idea behind cyclic pitch originates from helicopter control principles, where it was deemed essential in establishing controllable helicopter flight [3].

Since the 1960s several patents and research papers were published on the concept of collective and cyclic pitch control to propel and manoeuvre marine vehicles $[4,5,6,7,8,9,10,11]$. Despite showing great promise, the technology has not yet emerged as a viable and usable propulsion and manoeuvring system for AUVs for many different reasons. An example of a CCPP showing great potential, but not yet developed further and used in industry, is the propeller designed and built by [12] (rendering seen in Figure 1). Work on the CCPP developed by [13] was continued by several other researchers [14, 15, 16]. Observations were made by all researchers uncovering large discrepancies and results' uncertainty between the predicted and experimental performance of the CCPP. Recommendations were made on the need for the determination of more accurate hydrodynamic coefficients and increased insight into the flow behaviour on a detailed level, potentially through the use of advanced numerical methods and flow modelling techniques.

A follow-up research project was set-up, aimed at the extended analysis and future improvement of the hydrodynamic performance of the CCPP. The project uses Computational Fluid Dynamics (CFD) to investigate the complex and highly unsteady flow and force behaviour involved in the constant pitching of the propeller's blades. Numerical investigations provide some clear advantages and benefits when compared to more traditional experimental research, as discussed in the related earlier published work by the current author $[17,18,19]$. In the initial stage, a two-dimensional numerical model, relating the CCPP'S performance to that of pitching hydrofoils, was developed [17] and used as a preliminary performance analysis tool [19]. The twodimensional study investigated the CCPP under bollard pull condition (no forward velocity) and showed the importance of the applied collective pitch angle, and the resulting drag generation, on the effectiveness and efficiency of the manoeuvring force. For further analysis purposes, a three-dimensional methodology was developed based on the conclusions of the two-dimensional study [18]. The limitations of the two-dimensional method resulted in an inability to research the effects of the advance ratio and capture the complex three-dimensional flow behaviour. The developed three-dimensional showed great potential as a research tool for a more detailed investigation of the effect of different operational and design parameters on the CCPP's hydrodynamic 
performance. However, before such an investigation is possible additional verification and validation of the numerical methodology was identified as essential to ensure the produced results represent the system accurately and credibly. If one wants to use a numerical research methodology without running the risk of being misled by the results, proper consideration needs to be given to the numerical uncertainty and modelling errors.

In the current paper, the first stage of a three part evaluation of the numerical methodology is undertaken, with an analysis of the numerical uncertainty and parts two and three analysing and evaluating the validity and applicability of the numerical model. As said, first the numerical uncertainty is evaluated further in the current study. The aim of the study is to establish the total numerical uncertainty of the applied methodology, evaluating the effect of the oscillatory convergence and both the spatial and time discretisation. All three components need to be taken into account because of both the applied numerical procedure and the physical nature of the CCPP operation, in contrast to similar past studies where only a single or two of the components were studied simultaneously. Based on the quantified uncertainty, combined with analysis of the experimental uncertainty, the validity and applicability of the numerical model can be studied further. Furthermore, the developed numerical model then can be used for a more extensive analysis of the effect of operational conditions as well as design changes on the CCPP's hydrodynamic performance.

The paper is organised in the following manner. In Section 2 a introduction on the CCPP is presented, outlining and explaining relevant concepts. Section 3 discusses the research methodology, including the numerical model, the quantification of the hydrodynamic performance parameters to be used in the study, numerical uncertainty estimation methodology and the study parameters and cases to be investigated. In Section 4 and 5 the numerical uncertainty of the CFD-methodology is estimated and analysed, as is the main objective of the paper. Finally, Section 6 summarises the work done and provides insight into future work.

\section{The Collective and Cyclic Pitch Propeller}

\subsection{CCPP Characterisation}

The collective and cyclic pitch propeller (CCPP) applies two different pitch concepts to generate both propulsion and manoeuvring forces. For the current propeller, the pitch angle is defined as the angle between the blade 
chord and the $y z$-plane, with positive values with the leading edge orientated in positive $x$-direction (directions defined in Figure 1, more on propeller pitch found in work by [2]). Collective pitch governs the direction and magnitude of the propulsion force, while the cyclic pitch variation over the azimuthal cycle controls the generated manoeuvring force(s). The propulsion force is defined as the longitudinal force in $x$-direction and the manoeuvring forces are the forces perpendicular to the rotational axis, i.e. forces in the $y z$-plane.

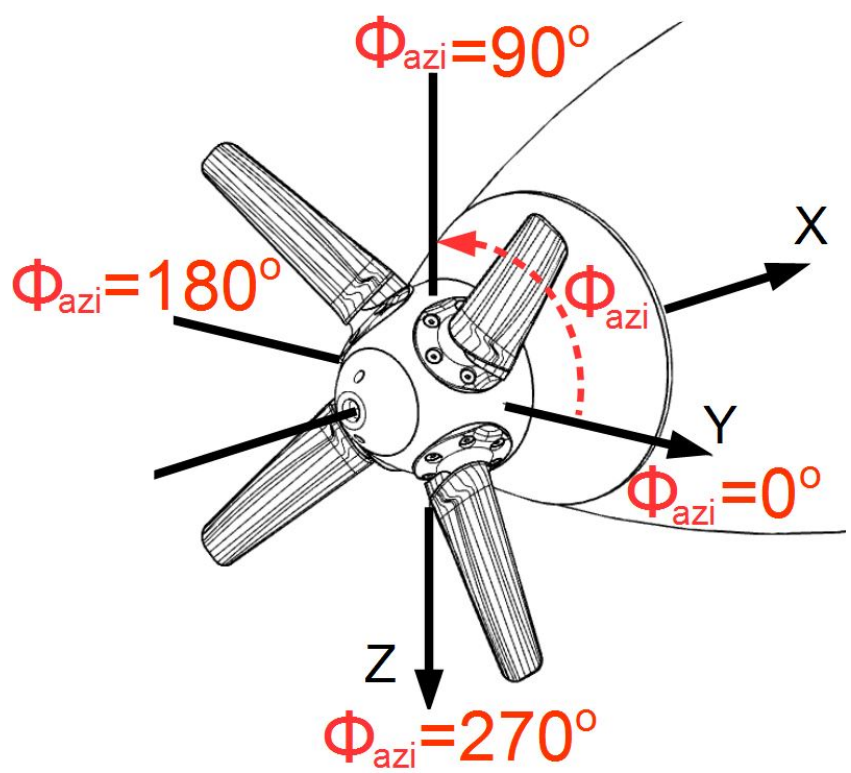

Figure 1: Definition of azimuthal cycle and axis orientation

The CCPP is intended to be fitted on a torpedo-shaped AUV and was designed with specific behavioural characteristics and geometry specifications, as discussed in previous work $[19,18]$ and an overview of the main characteristics presented in Table 2. One of the most important characteristics of the CCPP is the blade rake angle, i.e. the angle between the blade pitch axis and the propeller rotational plane (perpendicular to the rotational axis). The rake angle is responsible for a de-composition of the generated forces, allowing for the effective generation of a manoeuvring force. 
Table 2: CCPP geomtetry characteristics

\begin{tabular}{lll}
\hline \hline Number of blades & $N$ & 4 \\
\hline Blade section profile & - & NACA0012 \\
\hline Chord length (max.) & $c_{\max }$ & $0.037[\mathrm{~m}]$ \\
\hline Blade span & $s$ & $0.118[\mathrm{~m}]$ \\
\hline Blade span profile & - & Tapered to $0.70 c_{\max }$ \\
\hline Rake angle & $\beta$ & $20\left[^{\circ}\right]$ \\
\hline Diameter & $D$ & $0.305[\mathrm{~m}]$ \\
\hline Blade root radius & $r$ & $0.042[\mathrm{~m}]$ \\
\hline Blade area ratio & $A / A_{0}$ & $0.15[-]$ \\
\hline Pitch point & - & $0.25 c$ \\
\hline
\end{tabular}

\subsection{Collective and Cyclic Pitch Control}

The pitch motion of the CCPP is controlled through a single parameter for the collective pitch and two parameters for the cyclic pitch. In order for the CCPP to generate effective manoeuvring forces the relation between the applied blade pitch and the resulting manoeuvring force or side-force, is of great importance. Through cyclic pitch variation a force imbalance over the azimuthal cycle is generated. The force imbalance manifests itself in a pitching / yawing moment and consequently changes the AUV's trajectory in three degrees of freedom: surge, pitch and yaw.

The parameters controlling the pitch motion are combined in the pitch motion equation, seen in Eq. (1). The equation specifies the pitch angle of each individual blade $\Pi\left(\phi_{a z i}\right)$ as function of the blade's azimuthal position $\phi_{a z i}$. The prescribed collective pitch angle $\Pi_{\text {coll }}$ will determine the mean around which the pitch of each individual blade oscillates. Through a combination of the cyclic pitch parameters $\Pi_{c y c l}^{\mathfrak{1}} / \Pi_{c y c l}^{\leftrightarrow}$ both the amplitude and phase of the pitch oscillation over the azimuthal cycle is controlled.

$$
\Pi\left(\phi_{a z i}\right)=\Pi_{\text {coll }}+\quad \Pi_{c y c l}^{\uparrow} \cdot \sin \left(\phi_{a z i}+180^{\circ}\right)+\Pi_{c y c l}^{\leftrightarrow} \cdot \cos \left(\phi_{a z i}+180^{\circ}\right)
$$

A direct relation exists between the applied pitch angle, the generated propulsion and manoeuvring forces, and the resulting AUV motion. Currently, a one-to-one relation is assumed between the pitch angle and the 
resulting force / motion, as can be derived from the directional arrows included in the cyclic pitch parameter indices (referring to the intended AUV motion, as expected from the applied pitch profile). Unfortunately, both experimental and numerical CCPP research has shown that the relation is not as straightforward as outlined [14, 19, 18]. Depending on the applied operational conditions, a shift will occur between the orientation of the intended / expected side-force, and the resulting side-force because of the generation of a side-force component perpendicular to the intended side-force direction. The relation between the input and output will vary depending on the applied flow and pitch conditions and better understanding of the complex relation is the most important motivation of the current research.

\section{Methodology}

\subsection{Numerical Model Outline}

The numerical model under investigation was developed using ANSYS Fluent v16 [20] and is based on solving the unsteady RANS equations. An unsteady RANS-approach is chosen to provide a good balance between the ability to model highly unsteady flow phenomena, (dynamic) stall effects and model scale effects [21, 22, 23], and the computational cost involved. In addition, the k- $\omega$ SST transition turbulence model was chosen to provide closure to the equations. A PISO-algorithm was selected to achieve pressure-velocity coupling in the iterative solver. To realise spatial discretisation of the gradients a least-squares cell based method was used, with second-order upwind discretisation schemes for both the momentum and turbulence parameters. Time discretisation was achieved through a bounded second-order implicit dual-time stepping method.

The current model uses a periodic approach, in which only one blade of the CCPP is modelled in order to optimise the computational resource usage. A 90 degree modelling periodicity is established using a periodic boundary condition and allows to reduce the four-bladed system to a single blade model. Simulation of the dynamic blade motion is made possible by dividing the computational domain into two zones, as shown in Figure 2. An inner zone enables the simulation of the individual blade pitching, while the outer zone allows simulation of the rotation of the entire propeller. Through the chosen motion strategy, a fixed spatial grid approach can be used, which is less computationally expensive than a deforming mesh or re-meshing approach. 
The motion of both zones is enabled through a user-defined function, which implements a quaternion-based motion algorithm [18].

Through only modelling of the aft section of the AUV, a further limitation of the computational domain size is achieved. In Figure 3, the domain dimensions and defined boundaries are visualised. The different boundary conditions applied are a uniform velocity profile for the domain inlet, an outflow condition for the outlet, symmetry conditions for the top of the domain (to model a zero-shear slip wall), and a no-slip wall condition for the CCPP / AUV. The most important boundaries are the interface between the outer and inner zone, and the rotational periodic interface. Using a sliding interface boundary condition communication between both zones, and their non-conformal mesh surfaces, is ensured, while the periodic interface enables the periodic modelling strategy.

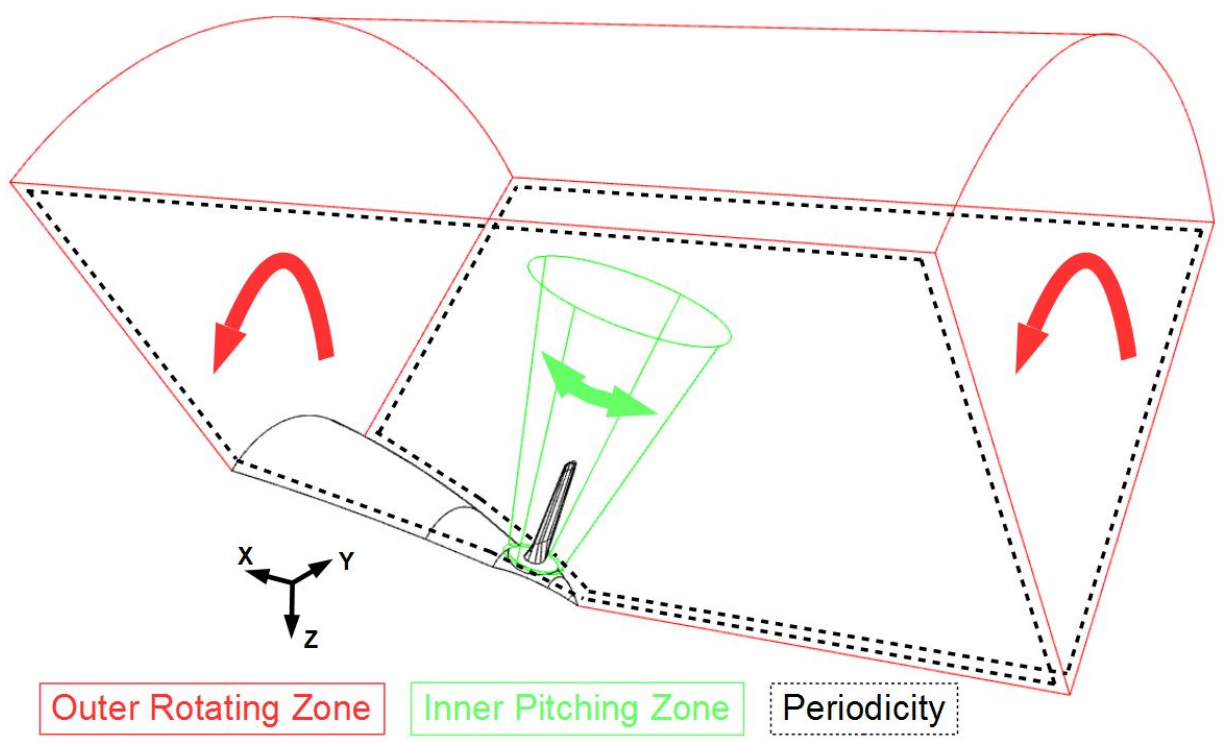

Figure 2: Numerical modelling strategy: periodic modelling and, separate rotational motion of AUV and pitching motion of the CCPP blade 


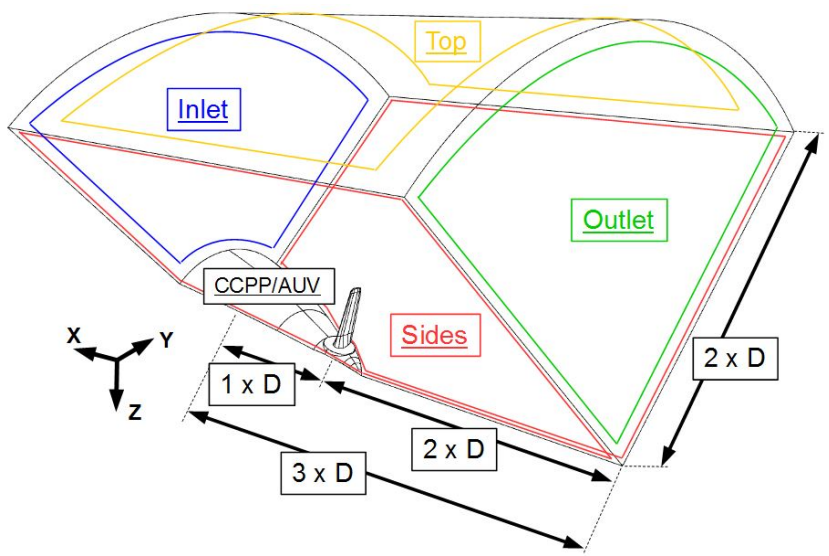

(a)

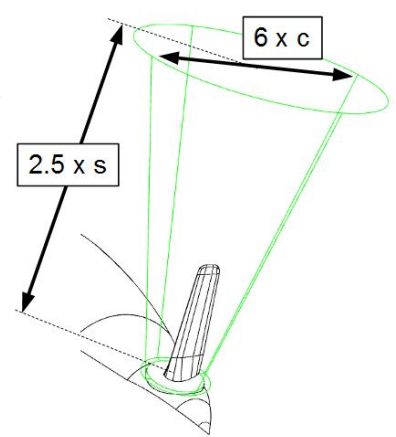

(b)

Figure 3: Computational domain dimensions and boundary definition for (a) the outer zone and (b) the inner zone

In order to numerically solve the governing flow equations, discretisation in both space and time is required. Spatial discretisation is achieved through the creation of a numerical grid, for which a multi-block structured hex grid approach is chosen a visualisation of the grid near the blade surface can be found in Figure 4). To allow for time discretisation, a time-step is selected based on the relevant flow phenomena and operational conditions. More discussion of both discretisation procedures, relevant selection of parameters, and parameters is added in Section 4. In order to establish the dependency of the numerical solution on the applied discretisation, verification is needed. Initial verification of the numerical methodology was based on the previously developed two-dimensional model, as reported by [18]. The study also reported on the first steps taken to provide insight into the validity of the generated numerical results. The conclusions showed that additional verification and validation is needed to completely evaluate the performance and capabilities of the developed numerical methodology. 

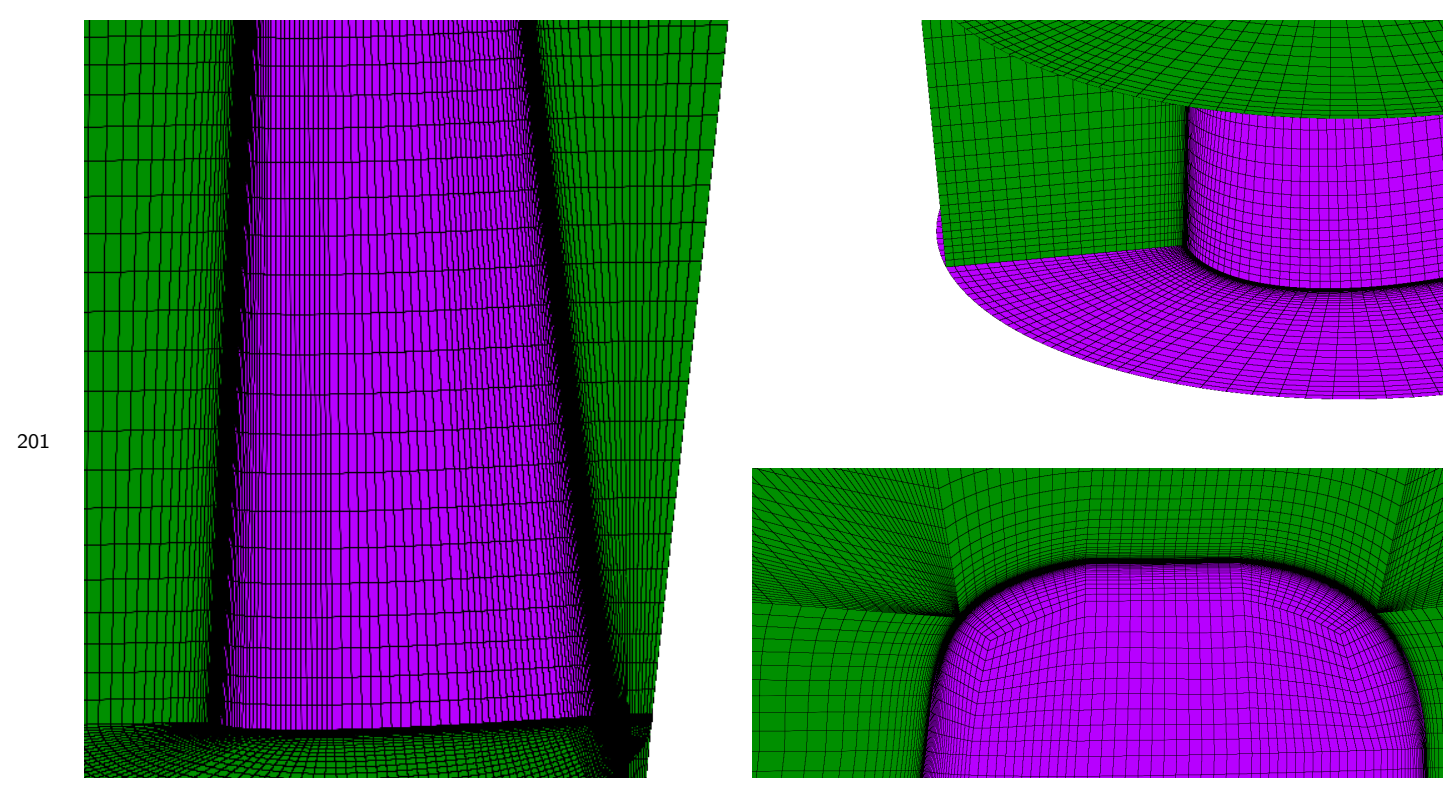

Figure 4: Spatial grid details (purple: on blade surface)

\subsection{Hydrodynamic Performance Quantification}

Although all resulting forces are of importance, the focus of the current research is the generation of an effective and efficient manoeuvring force. The CCPP's hydrodynamic performance is defined by the efficient creation of a usable side-force, and the uncertainty will thus be evaluated accordingly. Effectiveness is defined as the creation of a sufficiently large force to allow AUV manoeuvring, while efficiency refers to the ability to generate manoeuvring force in the required direction only.

The forces generated by the CCPP in the three principal directions are measured, processed and analysed. As discussed in Section 2.2, the chosen blade orientation, and intended force vectoring, is arbitrary and can simply be adjusted through the applied pitch profile. Since the numerical model is fully symmetric and periodic, a choice is made for one cyclic pitch setting (one side-force direction) to represent the full operational profile. In the current analysis, the cyclic pitch parameters were chosen in such a way that the intended side-force is expected to be a purely negative $y$-force. To eliminate confusion and bias of the chosen direction, the $y$ - and $z$-force components are referred to as the intended and perpendicular force components, respectively. The resulting side-force $\vec{F}_{s}$ is defined by the vector sum of the intended and perpendicular side-forces $\left(\vec{F}_{s}^{\text {int }}\right.$ and $\vec{F}_{s}^{\text {per }}$, respectively), as shown in Eq. (2). 
The force in $x$-direction is also analysed, as the CCPP's capabilities to generate a propulsion force $F_{p r}$ also need evaluation.

$$
\vec{F}_{s}=\vec{F}_{s}^{i n t}+\vec{F}_{s}^{p e r}
$$

The magnitude of the generated side-force determines its effectiveness, and is defined by Eq. (3). The magnitude-parameter $\left|F_{s}\right|$ gives a good indication of the usability of the side-force to effectively manoeuvre an AUV. The relation between the operational conditions, such as the flow velocity and the forward propulsion force, and the resulting side-force magnitude will be of major importance to establish the AUV's manoeuvring capabilities.

$$
\left|F_{s}\right|=\sqrt{\left(F_{s}^{\text {int }}\right)^{2}+\left(F_{s}^{p e r}\right)^{2}}
$$

As explained, the orientation of the generated side-force is as important as the force magnitude for the actual manoeuvring performance of the AUV. In the current study, the orientation of the side-force is used to establish the efficiency of the generated side-force. The parameter $\phi_{s}$, defined in Eq. (4), relates the azimuthal orientation of the resulting side-force (a function of the ratio between the forces in $y$ - and z-direction) to the intended azimuthal side-force orientation as determined by the ratio between the cyclic pitch parameters. Important to note is that the parameter, as defined here, is not an indication of the overall propeller efficiency but merely gives insight into the directional efficiency of the generated manoeuvring force.

$$
\phi_{s}=f\left(\Pi_{c y c l}^{\mathfrak{1}}: \Pi_{c y c l}^{\leftrightarrow} ; F_{s}^{\text {int }}: F_{s}^{p e r}\right)
$$

Both the side-force and propulsion force parameters are used to determine the performance of the numerical model and estimate the numerical uncertainty. In later stages of the research, a combination of the parameters, as well as additional yet to be defined parameters, are needed to investigate and evaluate the actual hydrodynamic performance and efficiency of the CCPP.

\subsection{Uncertainty Estimation}

As the use of CFD became common practice in the maritime and many other industries, the need for the establishment of quality assurance procedures and common practices arose. Within the application of numerical 
methods, the quality assurance procedures are often referred to as verification and validation. [24] stressed the importance of the differentiation between the numerical errors introduced by the techniques used to solve the selected equations (verification) and the inherent deficiencies of the applied mathematical models (validation). An extensive review of methods and procedures for verification and validation was done by [25] and provides the necessary background, ideas and nomenclature to develop an appropriate verification and validation methodology. Additionally, the International Towing Tank Committee specified practical guidelines and recommendations for the development, usage, and evaluation of the numerical and modelling uncertainty of CFD simulations for ship applications and marine hydrodynamics [26, 27].

The current work focuses on the verification aspect by estimation and analysis of the numerical uncertainty introduced by the developed methodology. [28] identified the discretisation error as the dominant component contributing to the numerical error and uncertainty. Through evaluation of the spatial and time discretisation, in addition to analysis of the uncertainty introduced by the periodic nature of the simulations, the total numerical uncertainty is estimated. By analysing all three outlined contributors to the numerical uncertainty, a comprehensive novel method is developed based on different earlier studies into single component studies to analyse the numerical uncertainty introduced by the developed methodology. The oscillatory uncertainty study, related to the periodic behaviour of the CCPP, is based on investigation of the difference in both mean and time-dependent force results between each rotation cycle. The spatial discretisation error can be determined via a grid refinement study on consistently refined grids [29]. A similar procedure, as described by [30] for the unsteady simulation of the launch environment of a space vehicle, should be applied for the time-discretisation error. Based on the subsequent results of the refined simulations, which should reduce the observed error, a power expansion as a function of the applied refinement will give an estimate of the discretisation error for a certain chosen discretisation level. By multiplying the error estimator by a safety factor [31], depending on the reliability of the power expansion fit, the numerical uncertainty is derived and estimated. The choice of discretisation level, for which the numerical uncertainty is evaluated, is established through an extended convergence analysis.

The entire procedure, i.e. convergence analysis and uncertainty estimation, is done for three operational cases, as discussed in the next sub-section, to cover a wide range of operational conditions and up to 6 refinement lev- 
els are used for the spatial and time discretisation. A final remark needs to be made concerning the fact that verification, and numerical uncertainty estimation, is a solely numerical procedure and does not requires any prior knowledge of experimental data. Once the numerical uncertainty is established simulation results can be compared to experimental data in a validation study to evaluate the numerical model's applicability and capability in simulation the outlined flow problem. An extensive validation study, and methodology evaluation, will be the scope of future work.

\subsection{Case Selection and Parameters}

The investigation into the numerical uncertainty of the numerical model evaluates the spatial and temporal discretisation procedure at a number of different operational conditions. Some parameters need a closer definition in order to be able to determine both the discretisation settings and operational conditions to be evaluated.

The operational conditions are defined by four parameters: the AUV's forward velocity $u$, the propeller's rotational speed $n$, the collective pitch angle $\Pi_{\text {coll }}$, and the cyclic pitch angle $\Pi_{c y c l}$. Three parameters are defined for the purpose of spatial discretisation. Through the chord-based parameter $n_{c}$, the blade span-based parameter $n_{s}$, and the inner cylinder radius-based parameter $n_{r}$, the discretisation in all three spatial directions can be defined. Finally, the time discretisation is based on a fraction of the rotational period $T$, defined as $T=\frac{60}{n}$.

Three conditions were selected to fully represent the CCPP's operational profile. Table 3 provides an overview of the selected cases, designated with the letters A, B and C for further referencing. Case A, under bollard pull (zero forward velocity), applies no collective pitch to focus on the side-force generation. Cases $\mathrm{B}$ and $\mathrm{C}$ are very similar and chosen to investigate both the influence of forward velocity, i.e. captive condition, and the effect of applying collective pitch. All selected conditions include some form of cyclic pitch, as this is what differentiates the CCPP and numerical model from other propellers and methodologies.

In total, four spatial discretisation levels were chosen to be evaluated, in combination with four time discretisation levels. Determination of the discretisation parameter was based on earlier two-dimensional work, as documented by [17]. Table 4 summarises the evaluated discretisation parameters and provides nomenclature for further referencing. The levels of the spatial discretisation are designed with a doubling of the entire cell count in mind 
(fine, coarse, and extra coarse mesh), with an extra level added to provide additional convergence resolution towards the finer mesh levels (medium). The different spatial discretisation levels are evaluated at the finest time discretisation level, as will be discussed in the results analysis. Table 4 shows that the time discretisation levels are defined by reducing the time-step by a factor of 2, similar to the total spatial cell count. The time-step discretisation is evaluated using the finest mesh discretisation, similar to the spatial discretisation process.

Table 3: Operational conditions case definition

\begin{tabular}{ccccc}
\hline \hline & $u[\mathrm{~m} / \mathrm{s}]$ & $n[\mathrm{rpm}]$ & $\Pi_{\text {coll }}\left[^{\circ}\right]$ & $\Pi_{\text {cycl }}\left[^{\circ}\right]$ \\
\cline { 2 - 5 } Case A & 0.0 & 400 & 0.0 & 20.0 \\
Case B & 1.2 & 300 & 0.0 & 10.0 \\
Case C & 1.2 & 300 & 15.0 & 10.0 \\
\hline
\end{tabular}

Table 4: Spatial / time discretisation case definition

\begin{tabular}{lcclllc}
\hline \hline \multicolumn{3}{c}{ Spatial } & & & \multicolumn{2}{c}{ Time } \\
\cline { 1 - 2 } \cline { 5 - 6 } Name & Dimension* & Cell count** & & Name & Dimension \\
\hline Extra Coarse & $54 \times 52 \times 36$ & $1.25 \mathrm{M}$ & & Extra Coarse & $T / 100$ \\
Coarse & $68 \times 66 \times 45$ & $2.50 \mathrm{M}$ & & Coarse & $T / 200$ \\
Medium & $79 \times 75 \times 52$ & $3.75 \mathrm{M}$ & & Medium & $T / 400$ \\
Fine & $86 \times 83 \times 57$ & $5.00 \mathrm{M}$ & & Fine & $T / 800$ \\
\hline
\end{tabular}

${ }^{*} n_{c} \times n_{s} \times n_{r}$

** $\mathrm{M}=$ million

\section{Convergence Analysis}

\subsection{Analysis Outline}

Solution convergence can be described as the arrival of the solution at a value that no longer changes with respect to a certain setting or parameter. In the current case, convergence is monitored to establish a converged, reference level to be used for the final uncertainty estimation and quantification. 
First of all, the oscillatory or periodic convergence of the solution is investigated to ensure every single simulated case can be evaluated further. Once oscillatory convergence is established for each separate case, the spatial and time convergence are investigated.

The convergence of the spatial and time discretisation is monitored and evaluated based on the propulsive force and both the intended and perpendicular force components. Both force time histories (including harmonic analysis using a Fast Fourier Transform) and mean force values are used in the convergence analysis. The cyclic force time histories are determined and plotted against the dimensionless time $T^{*}=\frac{t}{T}$ (with the propeller blade pitching up from its mean position as time starts).

\subsection{Oscillatory / Periodic Convergence}

The CCPP's operation is a periodic phenomenon, with one period defined by a single rotation of the entire propeller combined with a single pitch oscillation of each CCPP blade. As the blades oscillate over the cycle, so will the generated forces and moments. Therefore, the periodically averaged force / moment values are considered to converge, i.e. to become near constant over each subsequent rotational cycle. As such, the oscillatory or periodic convergence of each numerical simulation needs to be monitored to mitigate the influence of start-up effects and / or unwanted unsteady flow phenomena.

Based on the convergence of the different settings and cases, a number of observations can be made. For all the three cases, the convergence of the propulsive force is the slowest, requiring significantly more time than the other forces to reach oscillatory convergence. An example of the oscillatory convergence analysis is shown in Figure 5, for an indicative case, displaying the difference in convergence pace for the different force components. The 'instability' of the propulsive force convergence is the most obvious under bollard pull condition, where no real inflow into the propeller is present and numerical instabilities tend to not exit the numerical flow domain as easily. Additionally, a clear link between the convergence of the difference forces is present, when the propulsive force force convergence exhibits certain unsteady behaviour the side-force components tend to follow. A monitoring procedure was developed and used for all force components for all different settings and cases to ensure the solutions could be considered periodically converged. The averaged results of the last 10 cycles of each force component are to be used for further analysis and the actual uncertainty estimation. 


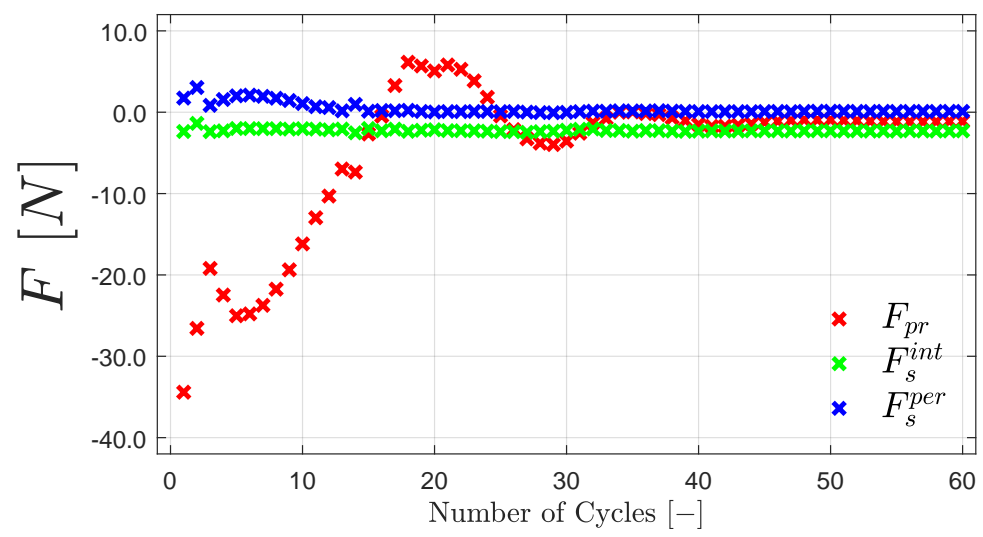

Figure 5: Oscillatory / periodic convergence evaluation

Two additional important conclusions were established based on the oscillatory convergence study results. Firstly, faster convergence was realised by starting at smaller time-step and then switching to the desired time-step after convergence of the coarse time-step was established. The results were verified by comparing the results from a case combing time-steps and a case fully simulated with the fine time-step. Secondly, it was concluded that starting from a fully converged case and adjusting certain operational settings to evaluate their influence also resulted in increased convergence speed of the new set case. All simulated cases will thus form an initialisation database for future cases and research to be simulated.

\subsection{Spatial Convergence}

The spatial convergence is investigated based on four grid levels to establish the dependence of the numerical solution on the applied grid resolution. Through the convergence analysis, a qualitative investigation of the influence of the spatial discretisation is performed. A quantitative analysis and complete error estimation based on data and results of the convergence analysis will be included in the next section.

Analysis of the convergence behaviour of the time histories highlights some important trends in the cyclic force behaviour. Figure 6 summarises 6 force components, illustrating the most significant trends observed in the spatial convergence. In general, good convergence is observed for the medium and fine spatial discretisation parameters for all three cases. The convergence shows a distinct phase difference occurring as the grid is refined (visible most clearly in Figure 6b, 6c, and 6e) and convergence of the overall amplitude 
behaviour of the time histories (seen in Figure 6a, 6b, 6c and 6e). The phase difference is visible in both the zero-force crossing behaviour, as well as in the location of the force extremes. Additionally, the occurrence and behaviour of minor peaks in the force time histories appears to converge well as the grid is refined (observed in Figure $6 \mathrm{~d}$ and $6 \mathrm{f}$ at $T^{*}=0.08$ ). Finally, minor differences between the fine and medium grid level cyclic force profile can be identified, e.g. in Figure 6c (at $\left.T^{*}=0.5\right)$. The observed trends are confirmed in the harmonics analysis, where the first order components converge for the medium and fine cases, as well as for the one-to-one relations between cases and components. Second and third order harmonics in the force components converged in a similar manner or were identified to not be of any significant magnitude.

Additional analysis of the spatial convergence behaviour is performed by calculating the mean force components of the time histories. Figure 7 shows all mean force components converging for the medium and fine grid case $(3.75 \mathrm{M}$ and $5.00 \mathrm{M}$ cells, respectively). The convergence for the mean side-force components for Cases B and C shows a converged solution for the coarse grid case $(2.50 \mathrm{M}$ cells). Furthermore, a clear convergence of the one-to-one relation of each case reciprocally for all mean force components is established, as well as of the mean force component relation within each case. For example, from Figure 7a, as the grid resolution increases, it becomes clear that the generated propulsive force is the largest for Case $\mathrm{C}$ and the smallest for Case A. 

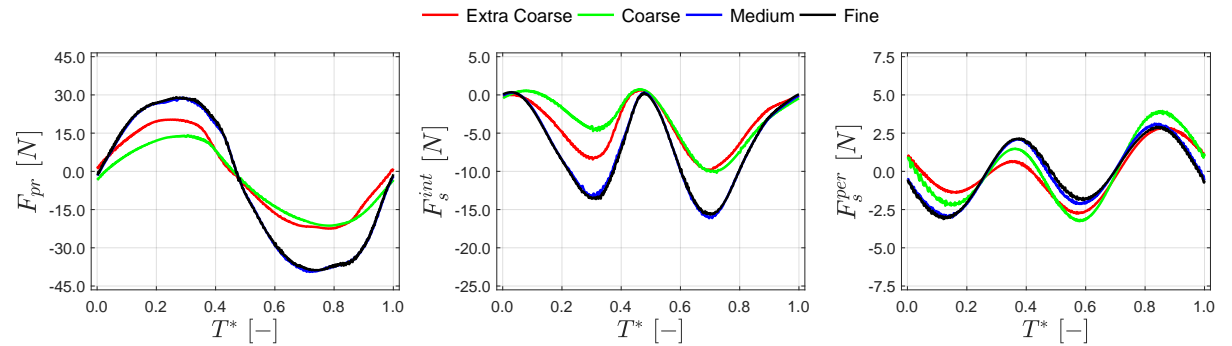

(a) Propulsive Force, Case A

(b) Intended Side-Force, Case A

(c) Perpendicular Side-Force, Case B
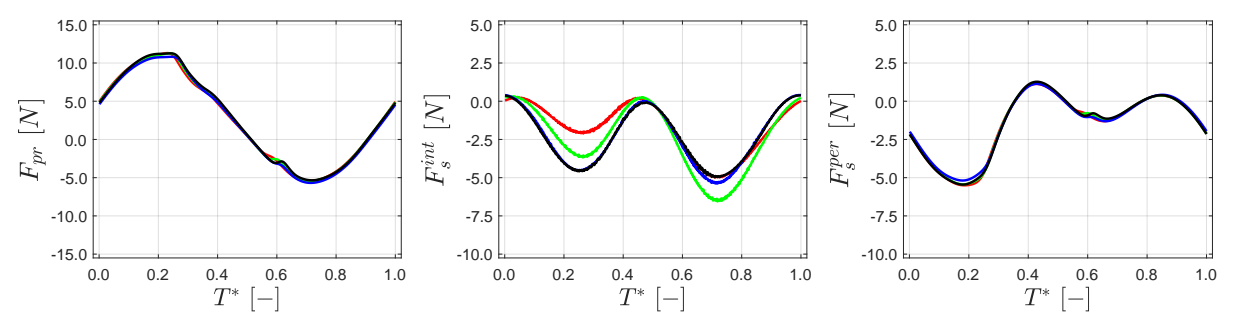

(d) Propulsive Force, Case C

(e) Intended Side-Force, Case B

(f) Perpendicular Side-Force, Case C

Figure 6: Spatial convergence evaluation of force time histories

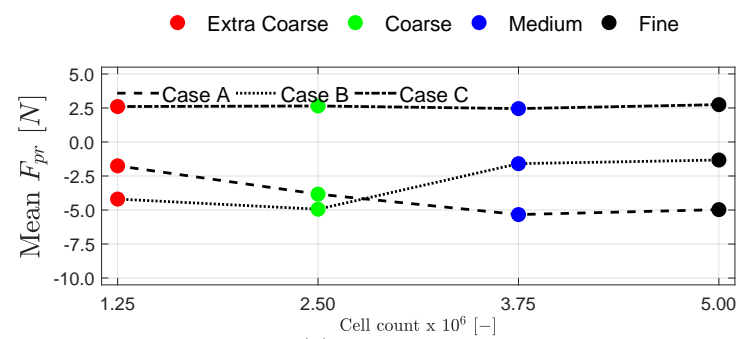

(a) Propulsive Force

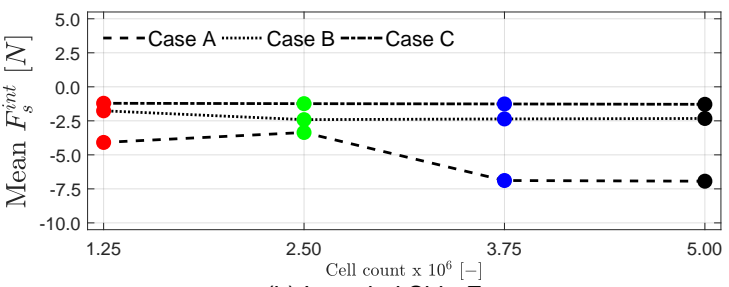

(b) Intended Side-Force

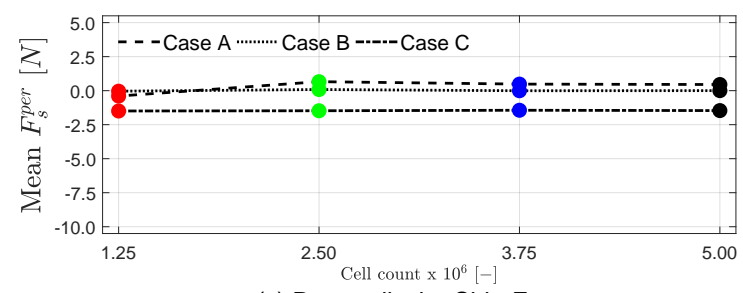

(c) Perpendicular Side-Force

Figure 7: Spatial convergence evaliation of mean force components 


\subsection{Time Convergence}

In a similar manner to the spatial convergence analysis, the dependence of the produced solution on the chosen time-step is examined. Two additional simulations were completed for Case A (T/600 and T/1000), as the initial results showed the need for added discretisation resolution to adequately assess the convergence behaviour. The time convergence results are plotted against the time-step percentage of the period to provide accurate insight into the time dependence of the simulations and thus the numerical methodology.

Again, only a selected number of time histories is plotted in Figure 8. All force components for Case A can be found in Figures 8a, 8b and 8c, while for Case B only the intended force component is shown and for Case $\mathrm{C}$ the propulsive and perpendicular force components are plotted. Case B and C display similar convergence behaviour for all force components, justifying the choice to not show all for both cases. Case A, which is the bollard pull condition, shows clear convergence issues and complications for all force components as force histories seem to change irregularly as the time-step is refined. The issues are present in all three force components and are assumed to originate from numerical instabilities remaining in the flow, circulating around the propeller and not being cleared out of the numerical domain by a physical flow as they do in Cases B and C (briefly discussed before). Figures $9 \mathrm{~d}, 9 \mathrm{e}$ and $9 \mathrm{f}$ do show good convergence behaviour for Case B and $\mathrm{C}$ with clear trends able to be distinguished for all force components. The finest time-step cases show very similar and thus converged behaviour of both large scale features, i.e. the amplitude and overall trend of the force history, as well as small scale features such as the minor humps (at $T^{*}=0.6$ in Figures $8 \mathrm{~d}$ and $\mathrm{f}$ ) and the exact phasing of the force extrema. Harmonic analysis once again established the same trends, with Case A showing irregular behaviour for all the first and second order component, while Case B and C show near time-step independence for all harmonics.

The observed trends in the time histories are confirmed in the mean force components, seen in Figure 9, showing convergence issues for Case A and good convergence for Cases $\mathrm{B}$ and $\mathrm{C}$. The mean of the propulsive and perpendicular side-force components of Case A changes quite drastically as the time-step is refined, while the intended side-force component remains constant until the finest time-steps. Nevertheless, the finest time-steps ( $T / 800$ and $T / 1000$ ) establish a converged one-to-one relationships between the different force components of each case reciprocally. The mean force components of Cases B and C confirm the near time-step independence of the solu- 

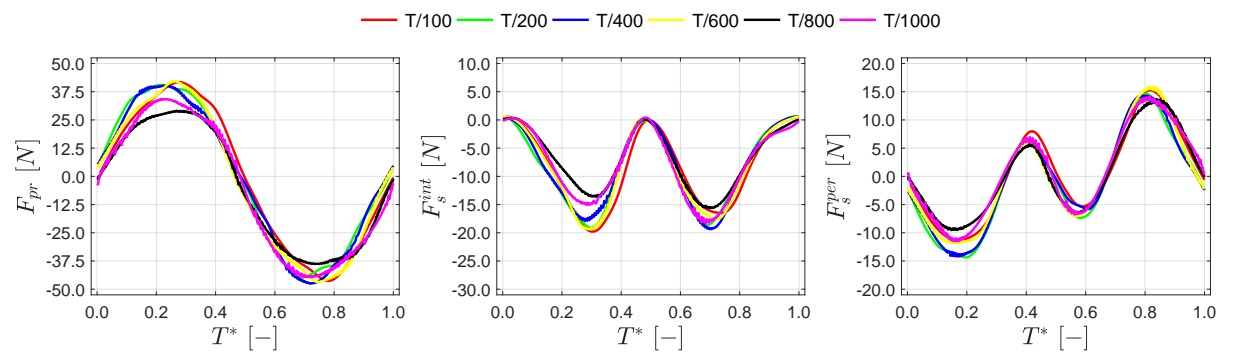

(a) Propulsive Force, Case A

(b) Intended Side-Force, Case A

(c) Perpendicular Side-Force, Case A
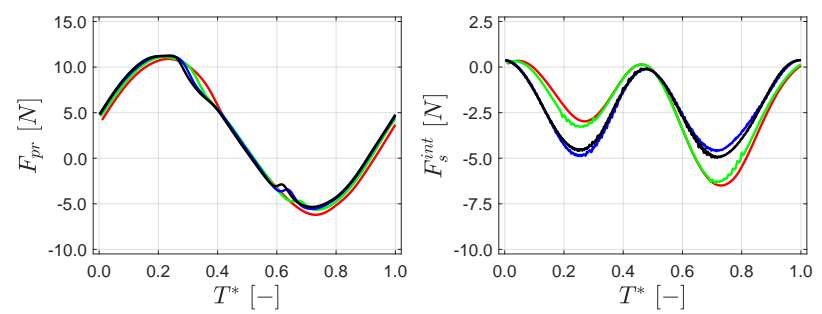

(d) Propulsive Force, Case C

(e) Intended Side-Force, Case B

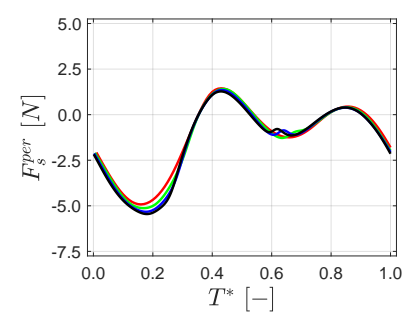

(f) Perpendicular Side-Force, Case C

Figure 8: Time convergence evaluation of force time histories

tions. Only the propulsive force component, best seen in Case B, appears to exhibit slower convergence, with convergence only established at the smallest time-steps. The side-force components show convergence of the mean force, and thus solution independence, over all simulated time-steps. 


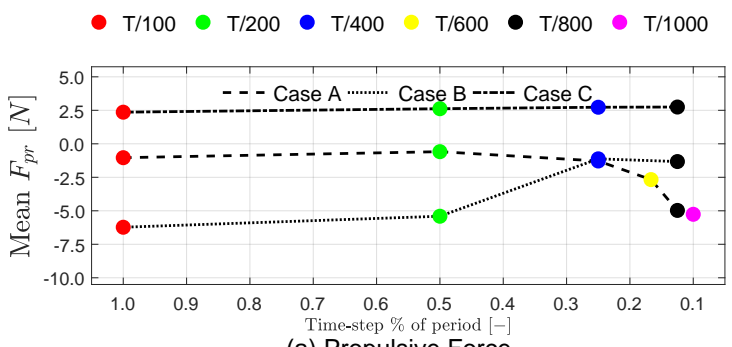

(a) Propulsive Force

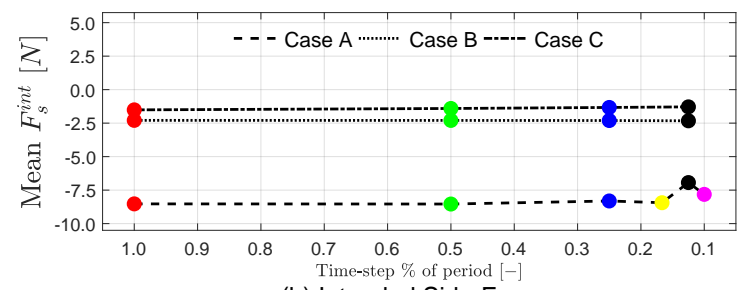

(b) Intended Side-Force

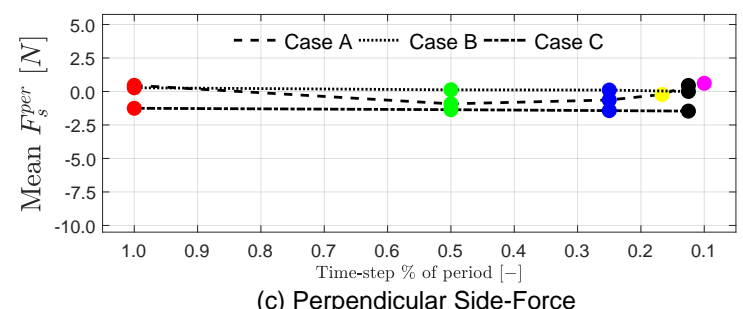

Figure 9: Time convergence evaluation of mean force components

\subsection{Summary Convergence Analysis}

The convergence analysis results in themselves do not provide quantitative insight into the numerical uncertainty. Nevertheless, key observations and conclusions are made based on the convergence analysis results and allow for the actual uncertainty estimation in the next section.

Based on the results of the convergence study, ten cycles is shown to be enough to consider the results oscillatory converged. Ten cycles considered will in turn be used to judge the contribution of the oscillatory behaviour to the total uncertainty. The spatial convergence shows the finest mesh level to be sufficiently converged for further analysis and will thus be used as the arbitrary level to evaluate the numerical uncertainty. The same applies to the second finest time discretisation level (finest for Cases B and C) in relation to the time convergence. Through proper selection of the discretisation levels, based on the convergence analysis, the numerical uncertainty is limited in essence through understanding that the solution no longer changes as the 
discretisation is refined, i.e. a discretisation-independent solution is determined. Based on the convergence analysis, final answers on the numerical uncertainty introduced by the developed numerical methodology can finally be answered.

A last conclusion based on the convergence results relates to any and all future work undertaken using the developed numerical methodology. The selected discretion levels will be used as main settings and parameters for any and all future simulations, including the planned validation and model evaluation study and further analyses into the CCPP's hydrodynamic performance analysis.

\section{Uncertainty Estimation}

\subsection{Estimation Procedure}

A procedure has to be established in order to estimate the numerical uncertainty of a specific simulation case. The numerical uncertainty $U_{\Gamma}$ is determined for an arbitrary flow variable or quantity $\Gamma$ and defines a range that contains the exact solution $\Gamma_{\text {exact }}$ of the chosen flow variable. Eq. (5) shows that the uncertainty $U_{\Gamma}\left(\Gamma_{i}\right)$ is a function of a specific arbitrary flow variable $\Gamma_{i}$, which is calculated for a certain case and discretisation level (as indicated by the sub-script ' $i$ ').

$$
\Gamma_{i}-U_{\Gamma}\left(\Gamma_{i}\right) \leq \Gamma_{\text {exact }} \leq \Gamma_{i}+U_{\Gamma}\left(\Gamma_{i}\right)
$$

The values of the arbitrary flow variable(s) are a direct result of any flow simulation, the numerical uncertainty itself however has to be calculated. In the current work, the uncertainty is presumed to be influenced by three factors: the oscillatory behaviour of the solution, the spatial discretisation, and the time discretisation. The total uncertainty $U_{\Gamma}\left(\Gamma_{i}\right)$ is considered to be the vector sum of the oscillatory uncertainty $U_{\Gamma}^{\mathrm{osc}}\left(\Gamma_{i}\right)$, the spatial discretisation uncertainty $U_{\Gamma}^{\text {spat }}\left(\Gamma_{i}\right)$ and the time discretisation uncertainty $U_{\Gamma}^{\text {time }}\left(\Gamma_{i}\right)$, as seen in Eq. (6) (as determined for a specific case and discretisation level).

$$
U_{\Gamma}\left(\Gamma_{i}\right)=\sqrt{\left(U_{\Gamma}^{\mathrm{osc}}\right)^{2}+\left(U_{\Gamma}^{\mathrm{spat}}\right)^{2}+\left(U_{\Gamma}^{\text {time }}\right)^{2}}
$$

The oscillatory uncertainty is calculated based on the results of a number of simulated cycles $n_{\text {osc }}$ and determined to be a function of the standard 
21

deviation $\sigma_{\text {osc }}\left(\Gamma_{i}\right)$, as seen defined in full in Eq. (7). The number of oscillation cycles is chosen to be 10, as discussed in Section 5.2, and the mean value over those cycles $\overline{\Gamma_{i}}$ is equal to the actual value used for calculation and evaluation of the numerical uncertainty. Figure 10 illustrates the applied oscillatory uncertainty estimation procedure and provides further insight into the most important parameters in Eq. (7).

$$
\begin{aligned}
U_{\Gamma}^{\mathrm{osc}}\left(\Gamma_{i}\right) & =\frac{\sigma_{\mathrm{osc}}\left(\Gamma_{i}\right)}{\sqrt{n_{\mathrm{osc}}}} \\
\text { with } \quad \sigma_{\mathrm{osc}}\left(\Gamma_{i}\right) & =\sqrt{\frac{\sum_{j=1}^{n_{\mathrm{osc}}}\left(\Gamma_{i, j}-\overline{\Gamma_{i}}\right)^{2}}{n_{\mathrm{osc}}-1}}
\end{aligned}
$$

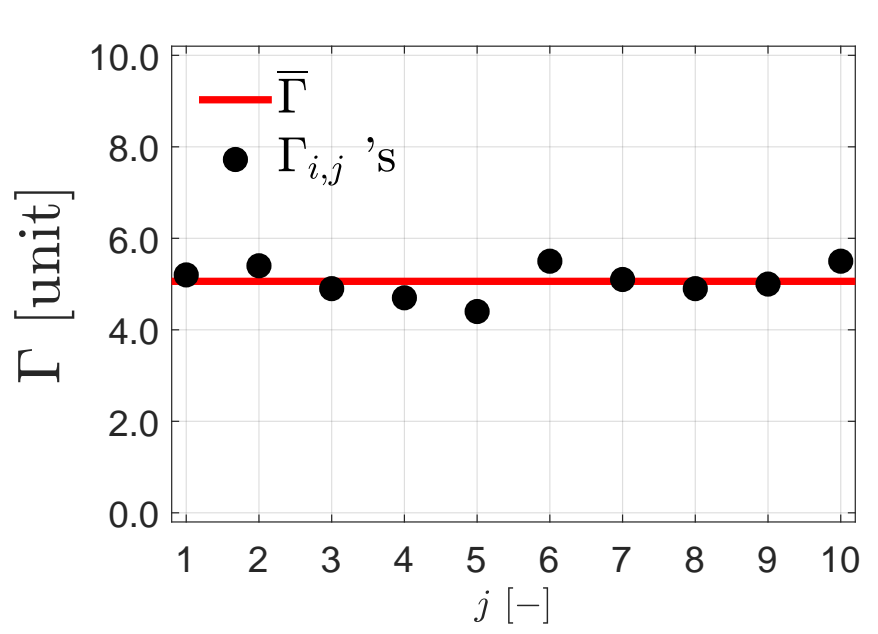

Figure 10: Oscillatory uncertainty estimation procedure

A more complex approach and procedure is required for the calculation of the uncertainty originating from the spatial and time discretisation. The procedure is the same for both space and time but is performed for each discretisation separately. The determination of the discretisation uncertainty is based on the systematic refinement of both the space and time discretisation. Through the regression of the systematic results, the discretisation uncertainty can be quantified. The regression fits a curve through the systematic refined results and allows for the numerical uncertainty to be calculated for an arbitrary discretisation level (indicated by $\Gamma_{i}$ ) based on the discretisation error $\epsilon_{\Gamma}\left(\Gamma_{i}\right)$ and a chosen / calculated safety factor $S F$. 


$$
U_{\Gamma}^{\text {spat }}\left(\Gamma_{i}\right) \quad / \quad U_{\Gamma}^{\mathrm{time}}\left(\Gamma_{i}\right)=S F \cdot \epsilon_{\Gamma}\left(\Gamma_{i}\right)
$$

The discretisation error is approximated by the difference $\delta_{\Gamma}\left(\Gamma_{i}\right)$ between the 'discretisation-less' solution $\Gamma_{0}$ and the chosen arbitrary discretisation level $\Gamma_{i}$, as shown in Eq. (9). However, the 'discretisation-less' solution is unknown and thus a further error estimation is needed. In the current research, the chosen error estimator is $\kappa \cdot h_{i}^{2}$, in which $\kappa$ is a constant to be determined and $h_{i}$ is the discretisation parameter related to a certain level of discretisation refinement in either space or time. The error estimator's assumed order, and thus the assumed order of convergence in both space and time, is presumed to be of the same order of the applied numerical methods (as discussed in Section 3.1).

$$
\epsilon_{\Gamma}\left(\Gamma_{i}\right) \simeq \delta_{\Gamma}\left(\Gamma_{i}\right)=\Gamma_{i}-\Gamma_{0}=\kappa \cdot h_{i}^{2}
$$

Eq. (9) can be considered in a least-squares sense in order to determine the two unknown variables, being the 'discretisation-less' solution $\Gamma_{0}$ and the constant $\kappa$, by solving a minimisation problem. The minimisation problem leads to a system of linear equations by using the systematically refined simulation results (with the number of linear equations equal to the number of refinement levels taken into account $n_{\mathrm{st}}$ ). To solve the system of linear equations, a weighted approach is chosen in which the results from 'finer' discretisation levels are given a higher weight in the regression (as can be found discussed in the work of [28]). Finally, the system is solved to determine both the unknowns, i.e. the 'discretisation-less' solution $\Gamma_{0}$ and the constant $\kappa$.

Rewriting of Eq. (9) allows the plotting of the fitted curve (i.e. the regression result) against the data used to determine it, as shown in Figure 11. The quality of the fit can be expressed through the standard deviation of the fitted curve $\sigma_{\text {fit }}$, and the difference between the actual simulation result and the fitted result $\Delta \Gamma_{\text {fit }}\left(\Gamma_{i}\right)$, defined in Eq. (10) and (11), respectively. The standard deviation is used as a measurement and indication of how good the fit is and in essence quantifies the quality of the error estimation. The difference between the simulation result and the fit further specifies the actual uncertainty of the selected discretisation level. Both parameters will be used to determine the appropriate safety factor and establish the total uncertainty associated with the spatial and time discretisation. 


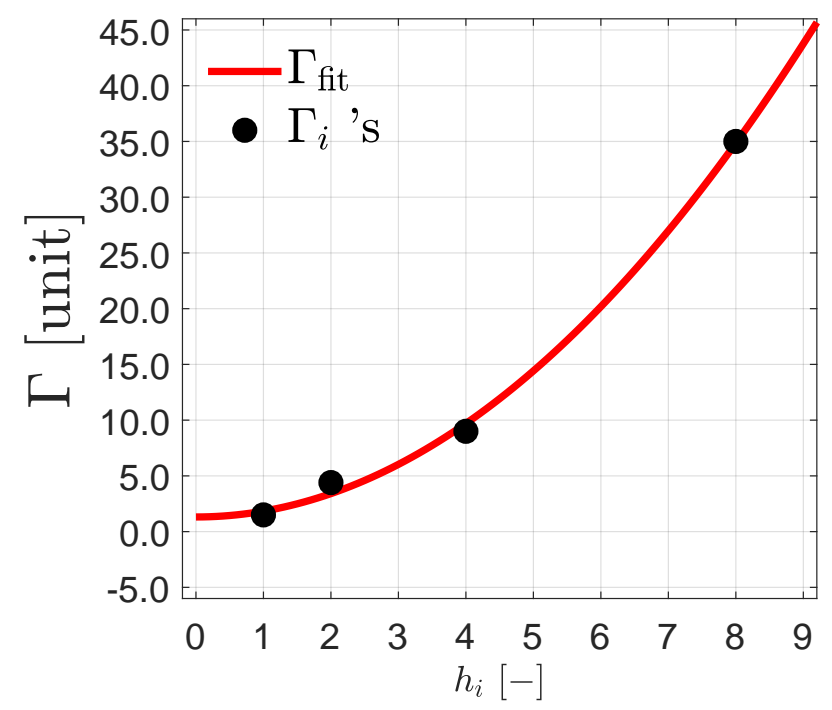

Figure 11: Time / space uncertainty estimation procedure

$$
\begin{gathered}
\sigma_{\mathrm{fit}}=\sqrt{\frac{\sum_{i=1}^{n_{\mathrm{st}}}\left(\Gamma_{i}-\Gamma_{\mathrm{fit}}\left(\Gamma_{i}\right)\right)^{2}}{n_{\mathrm{st}}-1}} \\
\Delta \Gamma_{\mathrm{fit}, i}=\left|\Gamma_{i}-\Gamma_{\mathrm{fit}}\left(\Gamma_{i}\right)\right|
\end{gathered}
$$

The safety factor (as specified in Eq. (8)) is defined to be equal to 1.25 when the estimation / fit is deemed reliable and 3.00 if not (following the work by [28] and the Grid Convergence Index procedure developed by [31]). In $\mathrm{Eq}(12)$, the procedure to determine the appropriate safety factor is outlined. Because the fit will always be perfect at $i=2$ an additional parameter is introduced to judge the reliability of the error estimation. The parameter $\Delta \Gamma_{\min }$ is defined as the difference between the calculated discretisation error and the finest simulated discretisation level.

$$
\begin{array}{lll}
\text { for } n_{s t}=2 & \text { and if } \epsilon_{\Gamma}>\Delta \Gamma_{\text {min }} & : S F=1.25 \\
\text { for } n_{s t}=3 / 4 / 6 & \text { else } & : S F=3.00 \\
& \text { and if } \sigma_{\text {fit }}<\Delta \Gamma_{\text {fit }, i} & : S F=1.25 \\
\text { else } & : S F=3.00
\end{array}
$$


The outlined uncertainty estimation procedure is applied to three flow variables; the propulsive force, the side-force magnitude, and the side-force orientation, thereby fully capturing the CCPP's hydrodynamic performance. As discussed before, the finest mesh and second finest time-step are used as reference and the actual results are the mean results for 10 oscillation cycles, for which the oscillatory uncertainty is determined $\left(n_{o s c}=10\right)$. The estimation of the uncertainty relating to the space and time discretisation is to established using a to be selected number of discretisation levels, of which an overview is presented in Table 5 (with the relevant size parameters, determined by each mesh size or time-step size relative to the selected reference discretisation level mesh size or time-step size).

Table 5: Discretisation level parameters

\begin{tabular}{cccccc}
\hline \hline & \multicolumn{2}{c}{ Space } & & \multicolumn{2}{c}{ Time } \\
\cline { 2 - 3 } \cline { 5 - 6 }$i$ & Name & $h_{i}$ & & Name & $h_{i}$ \\
\hline 1 & Fine & 1.00 & & Fine & 1.00 \\
2 & Medium & 1.33 & & Medium & 2.00 \\
3 & Coarse & 2.00 & & Coarse & 4.00 \\
4 & Extra Coarse & 4.00 & Extra Coarse & 8.00 \\
5 & - & - & Extra Fine & 0.80 \\
6 & - & - & Fine-Medium & 1.33 \\
\hline
\end{tabular}

A minimum of two levels has to be selected to be able to determine a regression of the results. The spatial and time discretisation uncertainty is evaluated for $n_{s t}=2, n_{s t}=3$, and $n_{s t}=4$ (or $n_{s t}=6$ for the time discretisation of Case A). Through visual representation of the regression and calculation of the different associated discretisation errors, the numerical uncertainties for each discretisation and performance parameter are determined in order to establish the combined total numerical uncertainty.

\subsection{Propulsive Force Uncertainty}

The main task of a marine propeller, with the CCPP being no exception, is the generation of an effective and efficient propulsive force. Even though the main focus of the current research is the side-force generated by the 


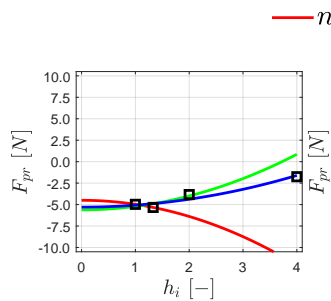

(a) Spat. Discr. Unc., Case A

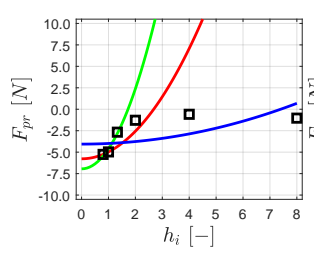

(e) Time Discr. Unc., Case A

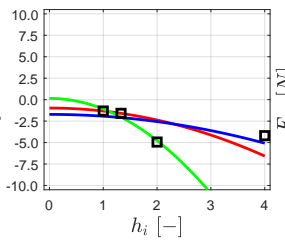

(b) Spat. Discr. Unc., Case B

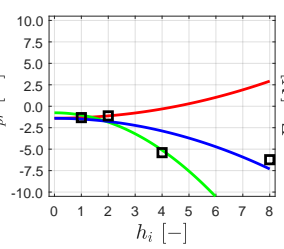

(f) Time Discr. Unc., Case B

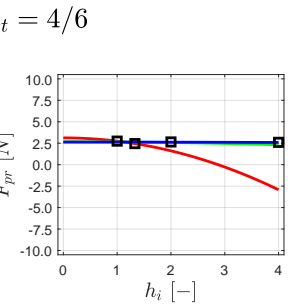

(c) Spat. Discr. Unc., Case C

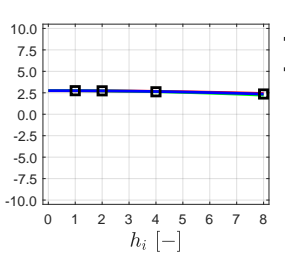

(g) Time Discr. Unc., Case C
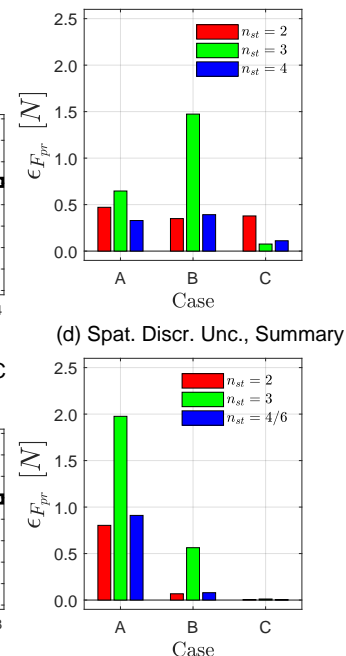

(h) Time Discr. Unc., Summary

Figure 12: Evaluation of the space and time discretisation error of the propulsive force.

CCPP, the evaluation of the performance of the propulsive force, and the effects of the side-force generation on it, is of great importance.

Before the total uncertainty of the propulsive force can be analysed and discussed, additional analysis of the discretisation regression results is needed. Figure 12 shows the regression analysis and calculated discretisation errors for both spatial and time discretisation for all three cases. From the results, it can be seen that the total error has a maximum value of $2.00[N]$, and for most cases / settings is below $1.00[N]$. Two extreme values can be distinguished, for Cases $\mathrm{A}$ and Case $\mathrm{B}$ at $n_{s t}=3$ in the time discretisation and spatial discretisation, respectively. The extreme values are related to the inability of the error estimator to address the observed convergence, i.e. the difference between the finest solutions (final $2 h_{i}$-values) is much smaller than the difference between the coarser and fine solution. A conservative approach dictates that the larger errors have to be taken into account in the further determination of the total numerical uncertainty, as will be discussed next. Therefore the uncertainty of each discretisation parameter is calculated through averaging of all uncertainty levels (after including the relevant safety factors per discretisation error).

A tabulated break-down of the total numerical uncertainty, based on the 
earlier drawn conclusions and the oscillatory uncertainty analysis, can be found in Table 6. The total numerical uncertainty is determined using Eq. (6) and the relative contributions are calculated through the projection of each contribution on the total uncertainty vector. No significant contribution to the error is observed for the oscillatory uncertainty component in any of the three cases. The highest total uncertainty is found in Case A, i.e. bollard pull, mainly because of the large time discretisation uncertainty, which is related back to the convergence issues discussed in the previous section. The difference between the total uncertainties in Cases $\mathrm{B}$ and $\mathrm{C}$ most likely originates from the applied collective pitch angle, with $\Pi_{\text {coll }}=0.0^{\circ}$ for Cases B and in Case $\mathrm{C} \Pi_{\text {coll }}=15.0^{\circ}$. As actual propulsion forces are intended to be generated, and the mean value of the propulsive force is expected to move away from zero, the uncertainty decreases. Additionally, the relative contributions show again a clear difference between bollard pull and captive cases. The total uncertainty of Case A is dominated by the time discretisation error, while the spatial discretisation clearly dictates the total uncertainty in the captive cases (Cases B and C). More general overall conclusions will be discussed at the end of the section, after analysis of the numerical uncertainty for the remaining two performance parameters.

Table 6: Uncertainty estimation break-down of the propulsive force $\left(\Gamma_{i}=F_{p r}\right)$

\begin{tabular}{|c|c|c|c|c|c|c|}
\hline \multirow{3}{*}{$\Gamma_{i}[N]$} & \multicolumn{2}{|c|}{ Case A } & \multicolumn{2}{|c|}{ Case B } & \multicolumn{2}{|c|}{ Case C } \\
\hline & \multicolumn{2}{|c|}{-4.97} & \multicolumn{2}{|c|}{-1.32} & \multicolumn{2}{|c|}{2.74} \\
\hline & Abs. $[N]$ & Rel. [\%] & Abs. $[N]$ & Rel. [\%] & Abs. $[N]$ & Rel. [\%] \\
\hline$U_{\Gamma}^{\mathrm{osc}}$ & \pm 0.02 & 0.0 & \pm 0.07 & 0.3 & \pm 0.00 & 0.0 \\
\hline$U_{\Gamma}^{\text {spat }}$ & \pm 0.60 & 7.8 & \pm 1.15 & 91.9 & \pm 0.34 & 99.9 \\
\hline$U_{\Gamma}^{\text {time }}$ & \pm 2.07 & 92.2 & \pm 0.34 & 7.8 & \pm 0.01 & 0.1 \\
\hline$U_{\Gamma}[N]$ & \multicolumn{2}{|c|}{ \pm 2.15} & \multicolumn{2}{|c|}{ \pm 1.20} & \multicolumn{2}{|c|}{ \pm 0.34} \\
\hline
\end{tabular}

\subsection{Side-Force Magnitude Uncertainty}

The effectiveness of the CCPP in manoeuvring an AUV will depend on the magnitude of the generated side-force. The side-force magnitude based on the force results presented before in the convergence analysis (through Eq. (3)). To analyse the total uncertainty of the side-force magnitude, the 


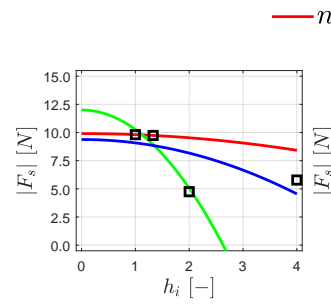

(a) Spat. Discr. Unc., Case A

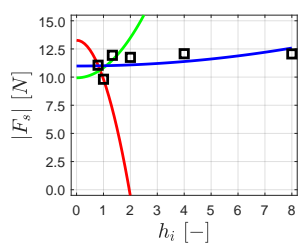

(e) Time Discr. Unc., Case A

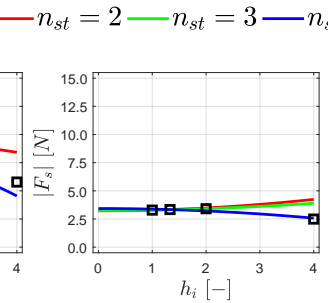

(b) Spat. Discr. Unc., Case B

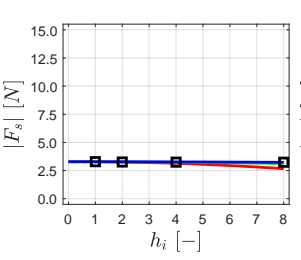

(f) Time Discr. Unc., Case B

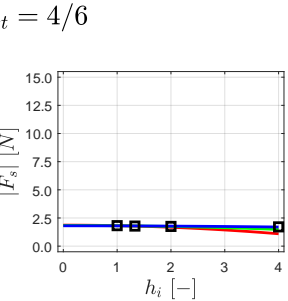

(c) Spat. Discr. Unc., Case $\mathrm{C}$

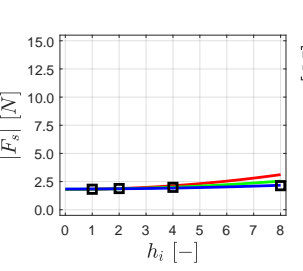

(g) Time Discr. Unc., Case C
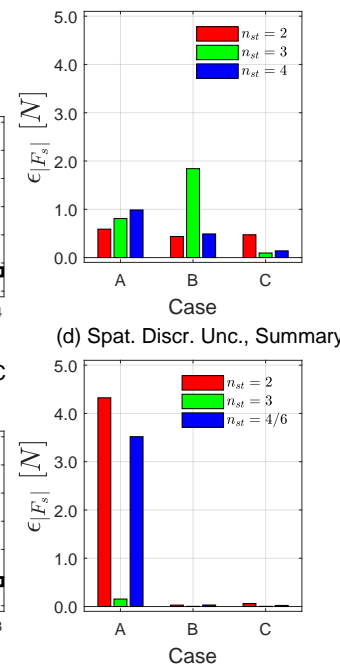

(h) Time Discr. Unc., Summary

Figure 13: Evaluation of the space and time discretisation error of the side-force magnitude.

same procedure as for the total uncertainty of the propulsive force is applied (both in calculation and plotting of the resulting uncertainties).

The regression analysis and related discretisation errors can be found plotted in Figure 13. An immediate observation can be made concerning the difference between Case $\mathrm{A}$ on the one hand and Cases B and $\mathrm{C}$ on the other hand. While the discretisation error is relatively small for Case B and C, as could already be expected based on the convergence analysis results, this is not the case for Case $\mathrm{A}$ (at various different values of $n_{s t}$ ). The discretisation error exceeds $2.00[N]$ in the spatial discretisation and even $4.00[N]$ in the case of the time discretisation. For the spatial discretisation, the discretisation error can be related to the same reason as discussed in the previous sub-section, i.e. the inability for the error estimator to capture the small difference between the finer solutions. The error of the spatial discretisation is directly related to the lack of convergence of the perpendicular forces, i.e. force in $y$ - and $z$-direction, as discussed in the convergence analysis. Combined, the convergence of both forces results in the observed error of the side-force magnitude predictions. Again, the conservative approach is chosen and all errors are taken into account to estimate the total uncertainty for each case. 
A tabulated overview of the total numerical uncertainty is presented in Table 7, determined using the previously presented results and conclusions. Similar conclusions as before can be drawn concerning the oscillatory uncertainty, as this contributes less than one percent to the total uncertainty. Case A shows the highest total uncertainty, as expected, resulting mainly from the high uncertainty in the time discretisation. The same trend seen in the previous sub-section is followed for Cases B and C, with no collective pitch being applied (Case B) resulting in a higher total uncertainty. Furthermore, it can once more be seen in the relative contributions that the total uncertainty for the captive cases is dominated by spatial discretisation uncertainty, while in the bollard pull case the time discretisation uncertainty is the main contributor to the total uncertainty.

Table 7: Uncertainty estimation break-down of the side-force magnitude $\left(\Gamma_{i}=\left|F_{s}\right|\right)$

\begin{tabular}{|c|c|c|c|c|c|c|}
\hline \multirow{3}{*}{$\Gamma_{i}[N]$} & \multicolumn{2}{|c|}{ Case A } & \multicolumn{2}{|c|}{ Case B } & \multicolumn{2}{|c|}{ Case C } \\
\hline & \multicolumn{2}{|c|}{9.81} & \multicolumn{2}{|c|}{3.29} & \multicolumn{2}{|c|}{1.82} \\
\hline & Abs. $[N]$ & Rel. [\%] & Abs. $[N]$ & Rel. [\%] & Abs. $[N]$ & Rel. [\%] \\
\hline$U_{\Gamma}^{\mathrm{osc}}$ & \pm 0.00 & 0.0 & \pm 0.01 & 0.0 & \pm 0.00 & 0.0 \\
\hline$U_{\Gamma}^{\text {spat }}$ & \pm 0.79 & 8.2 & \pm 0.92 & 99.9 & \pm 0.24 & 98.4 \\
\hline$U_{\Gamma}^{\text {time }}$ & \pm 2.67 & 91.8 & \pm 0.02 & 0.1 & \pm 0.03 & 1.6 \\
\hline$U_{\Gamma}[N]$ & \multicolumn{2}{|c|}{ \pm 2.78} & \multicolumn{2}{|c|}{ \pm 0.92} & \multicolumn{2}{|c|}{ \pm 0.24} \\
\hline
\end{tabular}

\subsection{Side-Force Orientation Uncertainty}

Finally, the total uncertainty in the prediction of the side-force orientation, used to evaluate the efficiency of the generated side-force, can be quantified. Again, the same procedure as the one outlined for the evaluation of the total uncertainty of both the propulsive force and the side-force magnitude (with the actual side-force orientation calculated using Eq. 4).

In Figure 14 the regression analysis for the side-force orientation is shown. The results are plotted in degrees and thus not represent force magnitudes as was the case for both earlier discussed performance parameters. Again a clear 


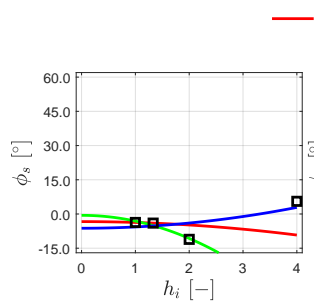

(a) Spat. Discr. Unc., Case A

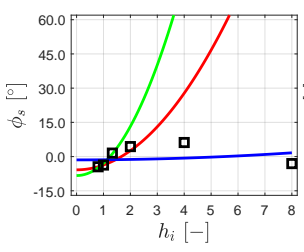

(e) Time Discr. Unc., Case A

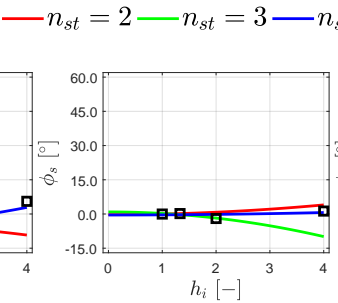

(b) Spat. Discr. Unc., Case B

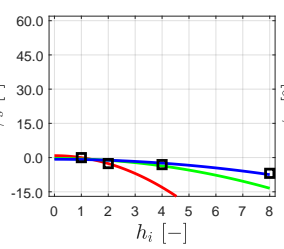

(f) Time Discr. Unc., Case B

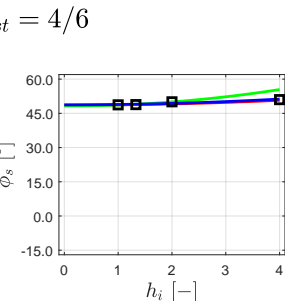

(c) Spat. Discr. Unc., Case C

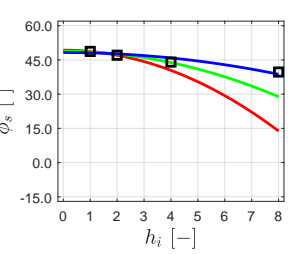

(g) Time Discr. Unc., Case C
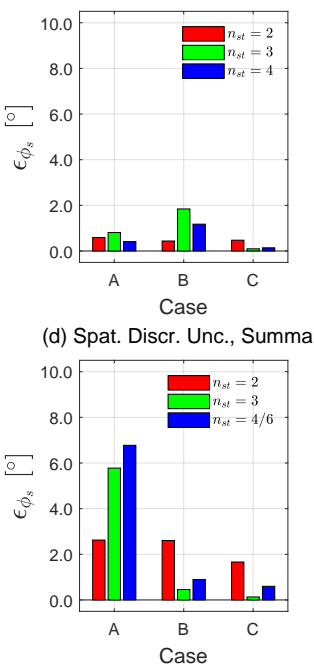

(h) Time Discr. Unc., Summary

Figure 14: Evaluation of the space and time discretisation error of the side-force orientation.

difference can be distinguished between the results of Case A and Case B/C. For Cases $\mathrm{B}$ and $\mathrm{C}$ the discretisation error is approx. $1.00\left[^{\circ}\right]$ for all cases, while the error exceeds $2.00\left[^{\circ}\right]$ for almost all cases within Case A, and is even as high as $6.00\left[^{\circ}\right]$ in the most extreme case. Despite good converge of the side-force orientation solutions for both time and space discretisation the discretisation error is relatively large because the error estimator again does not fully capture the convergence between the finest solutions.

A summary of the total numerical uncertainty for the side-force orientation is found in Table 8. As expected the same conclusions as for both other performance parameters concerning the oscillatory convergence can be drawn. No significant absolute values nor contribution to the total uncertainty is observed. The highest uncertainty is again found in Case A and the lowest in Case C. The relative contributions show a more convoluted picture, with the majority of the total uncertainty of all cases originating from the time discretisation but for Case B a significant contribution from the spatial discretisation uncertainty. 
Table 8: Uncertainty estimation break-down of the side-force orientation $\left(\Gamma_{i}=\phi_{s}\right)$

701

702

\begin{tabular}{|c|c|c|c|c|c|c|}
\hline \multirow{3}{*}{$\Gamma_{i}\left[^{\circ}\right]$} & \multicolumn{2}{|c|}{ Case A } & \multicolumn{2}{|c|}{ Case B } & \multicolumn{2}{|c|}{ Case C } \\
\hline & \multicolumn{2}{|c|}{-3.71} & \multicolumn{2}{|c|}{-0.03} & \multicolumn{2}{|c|}{48.73} \\
\hline & Abs. $\left[^{\circ}\right]$ & Rel. [\%] & Abs. $\left[^{\circ}\right]$ & Rel. [\%] & Abs. $\left[^{\circ}\right]$ & Rel. [\%] \\
\hline$U_{\Gamma}^{\mathrm{osc}}$ & \pm 0.07 & 0.0 & \pm 0.09 & 0.2 & \pm 0.04 & 0.2 \\
\hline$U_{\Gamma}^{\text {spat }}$ & \pm 0.60 & 1.4 & \pm 1.15 & 43.1 & \pm 0.24 & 8.0 \\
\hline$U_{\Gamma}^{\text {time }}$ & \pm 5.06 & 98.6 & \pm 1.32 & 56.6 & \pm 0.80 & 91.8 \\
\hline$U_{\Gamma}\left[^{\circ}\right]$ & \multicolumn{2}{|c|}{ \pm 5.10} & \multicolumn{2}{|c|}{ \pm 1.75} & \multicolumn{2}{|c|}{ \pm 0.83} \\
\hline
\end{tabular}

\subsection{Uncertainty Estimation Summary}

Based on the analysis of the uncertainty estimation of the three chosen performance variables a number of global observations and findings can be drawn. The observations and conclusions encompass discussion of the total uncertainty, the relative contributions from the different uncertainty contributors, and on how to apply the conclusions to further research and applications of the numerical model. Finally, a brief analysis of the physical implications of the results will be discussed.

The total uncertainty of the three performance parameters shows some clear trends and allows final conclusions on the uncertainty of the applied numerical methodology to be established. In combination with the results and conclusions of the convergence analysis, the numerical model is considered to be capable of producing converged results within a reasonable uncertainty for the captive cases (Cases B and C), as shown in Table 9. Based on the mean results (and associated total uncertainty) the produced results under bollard pull (Case A) have to be analysed with caution because of the inherent observed uncertainty. Together with the global results and convergence analysis, including the analysis of the harmonics, the potential of the developed model to investigate bollard pull conditions is shown but will require further investigation and development to reduce the associated numerical uncertainty. Future work will start with an extensive validation study and model evaluation to fully capture the model's applicability and in which the implications of the observed uncertainty will be considered in more detail for captive cases. 
Table 9: Uncertainty estimation results summary

\begin{tabular}{|c|c|c|c|c|c|c|}
\hline & \multicolumn{2}{|c|}{ Case A } & \multicolumn{2}{|c|}{ Case B } & \multicolumn{2}{|c|}{ Case C } \\
\hline & $\Gamma_{i}$ & $U_{\Gamma}$ & $\Gamma_{i}$ & $U_{\Gamma}$ & $\Gamma_{i}$ & $U_{\Gamma}$ \\
\hline$\left|F_{p r}\right|[N]$ & -4.97 & \pm 2.15 & -1.32 & \pm 1.20 & 2.74 & \pm 0.34 \\
\hline$\left|F_{s}\right|[N]$ & 9.81 & \pm 2.78 & 3.29 & \pm 0.92 & 1.82 & \pm 0.24 \\
\hline$\phi_{s}\left[^{\circ}\right]$ & -3.71 & \pm 5.10 & -0.03 & \pm 1.75 & 48.73 & \pm 0.83 \\
\hline
\end{tabular}

Additional findings can be drawn based on the observed relative contributions from Tables 6, 7 and 8. Overall, it can be concluded that for the force magnitude parameters (i.e. propulsive force and side-force magnitude) in the case of bollard pull, the time discretisation error contribution dominates the uncertainty, while in the captive cases the spatial discretisation error and uncertainty is dominant. A different observation needs to be made when looking at the side-force orientation results. The side-force orientation uncertainty of all cases becomes dominated by the time discretisation error. As the orientation of the side-force is all about timing of the forces over the azimuthal cycle the observed dependence on the chosen time-step and resulting uncertainty is considered an evident consequence.

The results of the current investigation into the uncertainty of the applied numerical model are of major importance for future research using the model, as well as in the potential further development of the model. For future simulation results, the total uncertainty will be calculated based on the actual oscillatory uncertainty of each specific case, and the established values for the space and time discretisation. Although the contribution of the oscillatory uncertainty was established to be minor, it will be analysed for every future case in order to ensure possible yet unknown implications are not disregarded. In future development of the numerical model, the bollard pull case will need additional investigation to try to reduce the observed uncertainty, i.e. increased results reliability, and enable more close-up and detailed analysis of these cases.

\section{Concluding Remarks}

The work presented in this paper has discussed the uncertainty estimation of a CFD-methodology developed to analyse the performance of a CCPP, a 
novel AUV propulsion and manoeuvring concept. The CFD-methodology [18] applies an unsteady RANS approach with the k- $\omega$ SST transition model to provide turbulence closure and second-order schemes applied to momentum, turbulence, and (implicit) time discretisation parameters. A periodic model, i.e. only one of four blades modelled, is realised to reduce the computational cost, while a sliding mesh is applied to enable the dynamic motion of both the entire propeller and the pitching blade itself.

An estimation of the numerical uncertainty was achieved through conducting a grid and time-step refinement study for three different operational conditions, in combination with an analysis of the oscillatory uncertainty introduced by the periodic nature of the simulations. Combining all three uncertainty components allowed to capture the uncertainty introduced in the current flow modelling approach in a comprehensive manner, addressing the different aspects and analysing their influence. A total of 26 cases were simulated with the subsequent results providing insight into the discretisation error, considered the dominant contributing component to the numerical error and uncertainty. The numerical uncertainty was estimated for three performance parameters: the generated propulsion force, the magnitude of side-force, and the orientation or phase-shift of the resulting side-force.

In order to achieve the final estimation of the uncertainty, a convergence analysis was conducted first. Through the convergence analysis it was shown that monitoring the oscillatory convergence is of importance and that as soon as the solution can be considered oscillatory converged 10 cycles suffice to evaluate the final result. The convergence analysis also showed that the finest applied space and time discretisation levels can be considered converged and be used as arbitrary results for the actual uncertainty estimation. The considered discretisation settings will be used for any and all future work into the CCPP's hydrodynamic performance undertaken using the numerical methodology.

The numerical uncertainty was estimated through the calculation of three parameters: the oscillatory uncertainty, the spatial discretisation uncertainty, and the time discretisation uncertainty. While the oscillatory uncertainty was determined to be minimal through analysis of the deviation of the mean value over 10 oscillatory cycles, a more complex procedure was applied to the spatial and time discretisation uncertainty estimation. Based on a leastsquares regression analysis, the discretisation error for both the spatial and time discretisation is determined. In combination with the calculation of a safety factor, the discretisation error established for all simulated cases, 
performance parameters, and conditions. In the case of the propulsive and side-force magnitude, the total uncertainty is dominated by the time discretisation uncertainty in the case of bollard pull conditions, while the total uncertainty of the captive cases is dominated by the spatial uncertainty. The total uncertainty in side-force orientation is observed to be almost fully a consequence of the time discretisation uncertainty for all simulated cases, which is considered an evident consequence as the orientation depends on the timing of the forces. Finally, it can be concluded that the total uncertainty for captive cases can be considered satisfactory for all performance parameters, while further work is needed to reduce the observed uncertainty of the simulations under bollard pull conditions.

Finally, a brief physical analysis of the simulated cases provides initial insight into the CCPP's performance. First of all, the results show that a negative thrust force is produced under both bollard pull and captive conditions at zero collective pitch, where it is expected for the CCPP to not produce a resulting thrust force. In earlier numerical work [19], using a two-dimensional methodology, the negative force was not observed and the current model confirms the assumption of the force generation being a threedimensional phenomenon. Second, the generation of a side-force can be seen in all cases, with the force increasing as the cyclic pitch angle is increased. Third and last, the previously documented (negative) influence of the collective pitch on the side-force orientation is re-confirmed. In essence, the model's potential in analysing the CCPP's hydrodynamic performance and the influence of different performance parameters is clearly visible, even when analysing these limited cases.

As the current work established the numerical uncertainty of the developed CFD-methodology, future work will incorporate two aspects. First, a further evaluation of the validity and applicability of the developed numerical model and, finally, the application of the methodology in the analysis and potential improvement of the hydrodynamic performance of the CCPP. The validation and model evaluation of the developed methodology will allow an investigation of the model's ability to capture and analyse the complex flow problem, as well as provide insight into future model adjustments and improvements. Additionally, the model evaluation will incorporate a critical reflection of the applicability and merits of the methodology. After validation and model evaluation, the methodology will be used to investigate the CCPP's hydrodynamic performance under different operational conditions. The focus of such an investigation will be understanding and improvement 
of the effectiveness and efficiency of the generated side-forces, represented by the side-force magnitude and orientation, respectively.

\section{References}

[1] K. Alam, T. Ray, S. G. Anavatti, A brief taxonomy of autonomous underwater vehicle design literature, Ocean Engineering 88 (2014) 627630.

[2] J. Carlton, Marine Propellers and Propulsion, 3rd Edition, ButterworthHeinemann, 2012.

[3] J. G. Leishman, Principles of Helicopter Aerodynamics, Cambridge University Press, 2000.

[4] Y.-H. B. Chen, S. K. Neely, K. A. Junghans, D. P. Bochinski, D. C. Robinson, A feasibility study of a novel propulsion system for unmanned underwater vehicles, in: UDT Europe 2008 symposium, Undersea Defence Technology Europe, Glasgow, UK, 2008.

[5] G. R. Gadefelt, Mechanism for the manoeuvring of propelled bodies through an homogeneous medium using multi-bladed propellers with adjustable blades, swedish Patent 171,641 - translated title (Aug. 3 1965).

[6] F. R. Haselton, Submarine hydrodynamic control system, uS Patent 3,101,066 (Aug. 20 1963).

[7] S. D. Jessup, Reduction of propeller vibration and cavitation by cyclic variation of blade pitch, Master's thesis, Massachusetts Institute of Technology (1976).

[8] W. Joosen, J. Van Manen, F. Van Der Walle, Large hub to diameter ratio propellers with programmed blade control, International Shipbuilding Progress 10 (101).

[9] B. Murray, J. Fraser, C. Dai, B. Maskew, Applications of cyclic pitch thrusters, in: Propellers/Shafting '94 Symposium, Society of Naval Architects and Marine Engineers, Virginia Beach, Virginia, 1994.

[10] E. J. Silberg, D. Everson, D. J. Haas, Marine propulsion-and-control system implementing articulated variable-pitch propellers, uS Patent 9,022,738 (May 5 2015). 
[11] J. L. Wham, L. A. Mackey, F. R. Haselton, Propeller system with electronically controlled cyclic and collective blade pitch, uS Patent 4,648,345 (Mar. 10 1987).

[12] T. C. Humphrey, N. Bose, C. Williams, M. Snow, Design and fabrication of a collective and cyclic pitch propeller, in: ASME 2004 23rd International Conference on Offshore Mechanics and Arctic Engineering, American Society of Mechanical Engineers, 2004, pp. 653-659.

[13] C. T. Humphrey, Design and fabrication of a collective and cyclic pitch propeller, Master's thesis, Memorial University of Newfoundland (2005).

[14] P. Niyomka, N. Bose, J. Binns, H. Nguyen, Experimental characterization of collective and cyclic pitch propulsion for underwater vehicle, in: The 3rd International Symposium on Marine Propulsors, Launceston, Tasmania, 2013, pp. 542-552.

[15] P. Niyomka, Performance and control of a collective and cyclic pitch propeller for an underwater vehicle, Ph.D. thesis, University of Tasmania - Australian Maritime College (2014).

[16] M. Q. Tran, S. A. T. P. Randeni, H. D. Nguyen, J. Binns, S. Chai, A. L. Forrest, Least squares optimisation algorithm based system identification of an autonomous underwater vehicle, in: Proceedings of the 3rd Vietnam Conference on Control and Automation, Vietnam, 2015.

[17] A. Dubois, Z. Q. Leong, H. D. Nguyen, J. R. Binns, Numerical modelling of the hydrodynamic performance of sinusoidally pitching hydrofoils, in: Proceedings of the 20th Australasian Fluid Mechanics Conference, Perth, Western Australia, Australia, 2016, pp. 1-4.

[18] A. Dubois, Z. Q. Leong, H. D. Nguyen, J. R. Binns, Development of a numerical model for the hydrodynamic performance analysis of a collective and cyclic pitch propeller, in: Proceedings of the Fifth International Symposium on Marine Propulsors - smp'17, 2017, pp. 203-210.

[19] A. Dubois, Z. Q. Leong, H. D. Nguyen, J. R. Binns, Hydrodynamic performance analysis of a collective and cyclic pitch propeller under bollard pull condition by numerical evaluation of two-dimensional pitching hydrofoils, Journal of Ship Research 62 (4). 
[20] I. ANSYS, ANSYS Fluent Theory Guide Release 16.0, SAS IP, Inc. (2015).

[21] S. Brizzolara, D. Villa, S. Gaggero, A systematic comparison between rans and panel methods for propeller analysis, in: Proceedings of 8th International Conference on Hydrodynamics, Nantes, France, 2008.

[22] V. Krasilnikov, J. Sun, K. H. Halse, CFD investigation in scale effect on propellers with different magnitude of skew in turbulent flow, in: The First International Symposium on Marine Propulsors, Trondheim, 2009, pp. 25-40.

[23] T. Watanabe, T. Kawamura, Y. Takekoshi, M. Maeda, S. H. Rhee, Simulation of steady and unsteady cavitation on a marine propeller using a RANS CFD code, in: Proceedings of the Fifth International Symposium on Cavitation, Osaka, Japan, 2003.

[24] L. Eça, M. Hoekstra, Verification and validation for marine applications of CFD, International Shipbuilding Progress 60 (1-4) (2013) 107-141.

[25] W. L. Oberkampf, T. G. Trucano, Verification and validation in computational fluid dynamics, Progress in Aerospace Sciences 38 (3) (2002) 209-272.

[26] ITTC, Ittc recommended procedures and guidelines - practical guidelines for ship CFD applications, Tech. rep. (2011).

[27] ITTC, Final report and recommendations to the 27th ITTC - specialist committee on CFD in marine hydrodynamics, Tech. rep. (2014).

[28] L. Eça, M. Hoekstra, A procedure for the estimation of the numerical uncertainty of CFD calculations based on grid refinement studies, Journal of Computational Physics 262 (2014) 104-130.

[29] NPARC Alliance, Tutorial on cfd verification and validation, https://www.grc.nasa.gov/www/wind/valid/tutorial/tutorial.html, accessed September, 2017 (2008).

[30] J. A. Housman, M. F. Barad, C. C. Kiris, Space-time accuracy assessment of CFD simulations for the launch environment, in: 29th AIAA Applied Aerodynamics Conference, 2011, p. 3650. 
${ }_{925}$ [31] P. Roache, Verification and validation in computational science and engineering. 